Magnetized electron emission from a small spherical dust grain in fusion related plasmas

A. Autricque, N. Fedorczak, S. A. Khrapak, L. Couëdel, B. Klumov, C. Arnas, N. Ning, J.-M. Layet, and C. Grisolia

Citation: Physics of Plasmas 24, 124502 (2017);

View online: https://doi.org/10.1063/1.4997695

View Table of Contents: http://aip.scitation.org/toc/php/24/12

Published by the American Institute of Physics

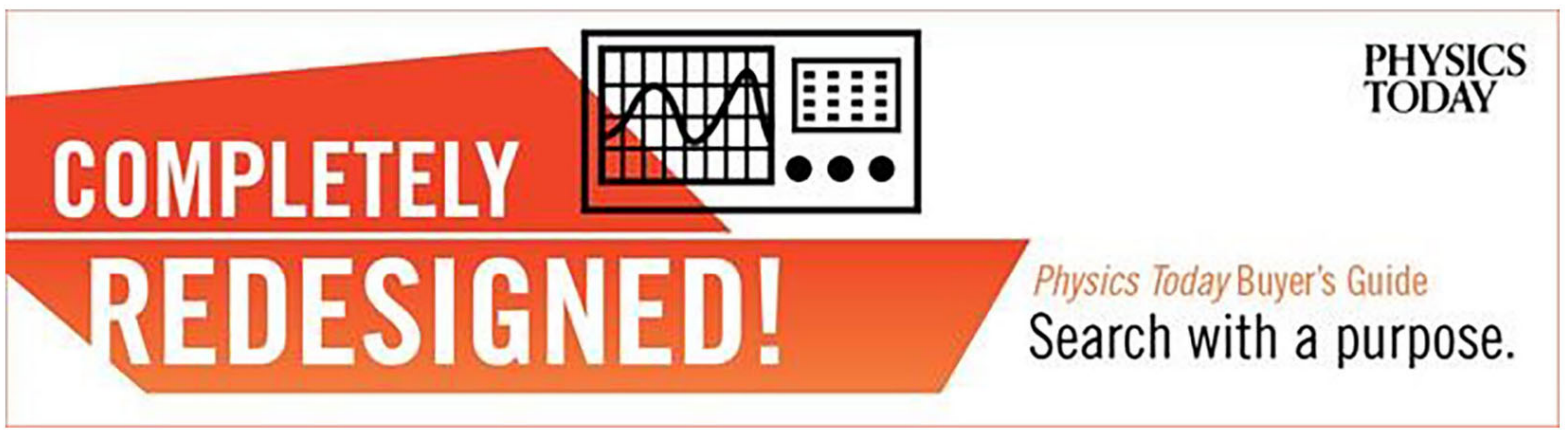




\title{
Magnetized electron emission from a small spherical dust grain in fusion related plasmas
}

\author{
A. Autricque, ${ }^{1, a)}$ N. Fedorczak, ${ }^{1}$ S. A. Khrapak, ${ }^{2,3}$ L. Couëdel, ${ }^{2}$ B. Klumov, ${ }^{2,4,5}$ C. Arnas, ${ }^{2}$ \\ N. Ning, ${ }^{2}$ J.-M. Layet, ${ }^{2,6}$ and C. Grisolia ${ }^{1,6}$ \\ ${ }^{1}$ CEA, IRFM, F-13108 Saint-Paul-Lez-Durance, France \\ ${ }^{2}$ Université Aix-Marseille, CNRS, UMR 7345, PIIM, 13397 Marseille CÉDEX 20, France \\ ${ }^{3}$ Institut für Materialphysik im Weltraum, Deutsches Zentrum für Luft- und Raumfahrt (DLR), \\ Oberpfaffenhofen, Germany \\ ${ }_{5}^{4}$ Joint Institute for High Temperatures, Russian Academy of Sciences, Moscow, Russia \\ ${ }^{5}$ Ural Federal University, Ekaterinburg, Russia \\ ${ }^{6}$ National Research Nuclear University “MEPhI," Moscow 115409, Russia
}

(Received 26 July 2017; accepted 9 November 2017; published online 6 December 2017)

The effect of magnetic field on the electron emission yield from a small spherical dust grain immersed in a plasma is investigated. It is demonstrated that, due to their gyromotion, some electrons can be promptly recollected on the grain's surface, which can reduce the emitted electron flux. The consequences are discussed mainly in the context of fusion-related applications, where the dust floating potential can be significantly reduced when positive while the dust lifetime remains weakly affected. In particular, we suggest that this effect should be included in the codes used to model the dust transport in tokamaks. Published by AIP Publishing. https://doi.org/10.1063/1.4997695

Plasma-wall interactions lead to the creation and mobilization of dust that will be a key issue for future fusion devices such as ITER. Upon interacting with the plasma, dust grains undergo erosion and release impurities that can reduce fusion performances by means of radiative losses and trigger plasma instabilities up to disruption. ${ }^{1}$

When immersed into a plasma, a dust grain charges negatively due to high electron mobility and a sheath forms in its vicinity, thus affecting plasma species collection, dust heating, and transport. Dust transport codes, such as DUMBO, ${ }^{2}$ MIGRAINe, ${ }^{3}$ DUSTT, ${ }^{4}$ DTOKS, ${ }^{5}$ or DUSTTRACK, ${ }^{6}$ are based on the Orbital Motion Limited (OML) ${ }^{7,8}$ theory to model dust charging. Several assumptions are made in the OML in order to come up with simple self-consistent expressions for the collection of plasma particles by a spherical dust grain: ${ }^{9}$ the surrounding plasma is assumed to be collisionless, unmagnetized, and the thick sheath regime is supposed to apply. These translate into constraints on the dust radius $r_{d}: r_{d} \ll \lambda_{e, i}, \rho_{L e, i}, \lambda_{D}$, where $\lambda_{e, i}$ is the electron (ion) mean free path, $\rho_{L e, i}$ is the electron (ion) Larmor radius, and $\lambda_{D}$ is the Debye length. Moreover, the energy and angular momentum conservation for an incoming plasma particle onto the conductive body are used as a starting point of the OML, which implies that barriers in the effective potential should be absent.

These constraining assumptions are already strongly challenged in tokamak Scrape-Off Layer (SOL) plasmas, where no grain larger than $\sim 10 \mu \mathrm{m}$ can be trustfully modeled, and where strong dust erosion can lead to high collisionality in its vicinity.

Electron emission from a dust grain can significantly alter its charge up to positive values. Since photoemission was found to be negligible in fusion applications, two electron emission processes are considered: secondary electron

\footnotetext{
${ }^{a}$ E-mail: adrien.autricque@cea.fr
}

emission (SEE) and thermionic emission (THE). ${ }^{10}$ Emitted electrons are subject to both the sheath potential profile and the magnetic field. The reduction of the electron emission yield from a positively charged grain has already been investigated and shown to be non-negligible, ${ }^{3}$ mainly because the mean energy of emitted electrons is of the order of the kinetic energy required to overcome the potential drop around the positive dust. The effect of the magnetic field has been investigated in the case of plasma collection by a spherical probe, ${ }^{11}$ but never in the case of electron emission in connection to dust transport codes in fusion related conditions. Emitted electrons have rather low temperatures, $\sim 1 \mathrm{eV}$, for SEE electrons and the dust surface temperature $T_{d}\left(\sim 10^{-2}-10^{-1} \mathrm{eV}\right)$ for THE electrons. ${ }^{12,13}$ Consequently, their gyroradii $\rho_{\text {Lse }}$ are one to two orders of magnitude smaller than that of typical SOL plasma electrons $\left(\rho_{L e} \gtrsim 1 \mu \mathrm{m}\right)$. Moreover, THE electrons are generally magnetized with respect to micron and sub-micron dust in standard SOL plasmas.

The gyromotion can lead to prompt recollection of emitted electrons on the dust surface regardless of their energy. This effect has been studied on Plasma-Facing Components (PFCs), considered as planar surfaces oriented with a given angle to the magnetic field (using Particle-In-Cell modelling, ${ }^{14}$ analytical models, ${ }^{15-17}$ and in experimental measurements ${ }^{18}$ ), but not in the case of a spherical emitting body, for which the ratio $r_{d} / \lambda_{D}$ might play an important role.

Since analytical expressions for the prompt recollection of emitted electrons around a spherical body in the presence of both electric and magnetic fields cannot be easily obtained, we propose to use a Monte Carlo approach in the particular case of THE, since it is the emission process that plays a more important role in dust floating potential calculations. ${ }^{4}$ Main results and effects on dust charging, transport, and heating are discussed for conditions relevant to fusionrelated plasmas. 
In the following paragraph, Monte Carlo simulations of the kinetics of thermionic electrons emitted by a spherical dust grain in the presence of a permanent and homogenous magnetic field $\mathbf{B}$ are presented.

In addition to $\mathbf{B}$, the other main factor leading to electron recollection is the sheath electric field $\mathbf{E}$, which depends largely on the dust surface potential $\phi_{d}$ and $\lambda_{D}$. Since the sheath potential profile around the dust particle is much steeper than typical SOL potential gradients, we choose $\mathbf{E}$ to have only a radial dependence: $\mathbf{E}=-\nabla \phi$, where $\phi$ is the Debye-Hückel potential

$$
\phi=\phi_{d} \frac{r_{d}}{r} \exp \left(-\frac{r-r_{d}}{\lambda_{D}}\right), \quad r \geq r_{d} .
$$

By choosing this potential profile, we assume that the potential wells are either absent or that their effects are negligible and the emitted electron population does not affect the potential profile (it remains fixed throughout the simulation). This hypotheses apply in two different situations: (i) the electron emission yield is low or (ii) the dust surface potential is close to zero. The first case is not of interest here since low electron emission will not affect the dust floating potential. At $\phi_{d} \sim 0$, potential wells can exist at high $T_{d}$, i.e., high THE yields. ${ }^{12,19}$ An estimation of the potential well depth $\phi_{\mathrm{vc}}$ and location $r_{\mathrm{vc}}$ can be done (within $15 \%$ accuracy) in the framework of the full Orbital Motion theory, using the results from Ref. 12, which are the only available but not certain estimates for the case under question. Calculations performed with the plasma parameters displayed in Table I and dust parameters detailed below show that $\left|e \phi_{\mathrm{vc}} / T_{e}\right| \sim 10^{-2}$. Estimating the electric field at the dust surface by $-\left(\phi_{d}-\phi_{\mathrm{vc}}\right) /\left(r_{d}-r_{\mathrm{vc}}\right)$ and the magnetic force by $B \sqrt{T_{d} / m_{e}}$, one finds that the magnetic force is about one order of magnitude higher than the electric force. Moreover, the electric force close to the grain is not significantly altered in the parameters ranges considered. On this ground, the potential well effects are not considered here. We plan to address this in future works.

The THE electron population is generated with random positions on the dust surface. The initial angle distribution function of THE electrons exit velocities is random. The velocity distribution is assumed to be Maxwellian with a temperature of $T_{d}$. The emitted electrons trajectories are computed using the forward Euler method until all of them have either reached the sheath edge given by the condition $r \geq 5 \lambda_{D}$ or have been recollected, i.e., $r \leq r_{d}$. All types of electron collisions are ignored.

The dust grain is assumed to be spherical and made of tungsten $(\mathrm{W})$, and the effects of eventual roughness at the

TABLE I. Typical SOL plasma parameters used in the simulations. The dust surface potential, radius, and temperature are varied for the parametric study shown in Fig. 3.

\begin{tabular}{lcc}
\hline \hline Name & Symbol & Value \\
\hline Electron temperature & $T_{\mathrm{e}}$ & $10 \mathrm{eV}$ \\
Background density & $n_{0}$ & $10^{20} \mathrm{~m}^{-3}$ \\
Debye length & $\lambda_{\mathrm{D}}$ & $2.4 \mu \mathrm{m}$ \\
Magnetic field & $B$ & $3 \mathrm{~T}$ \\
\hline \hline
\end{tabular}

surface are not considered. Thus, herein presented results can only be applied to spheroids such as solidified droplets and not to aggregated or flaky dust which has a more complex shape.

The dust parameters are varied for a parametric study in the following ranges: $0 \leq e \phi_{d} / T_{e} \leq 0.2, \quad r_{d} \leq 1 \mu \mathrm{m}$, and $3500 \mathrm{~K} \leq T_{d} \leq 6000 \mathrm{~K}$. Plasma parameters that are used in simulations and throughout this paper are summarized in Table I. They ensure that $r_{d}, \rho_{L s e} \ll \rho_{L e}$. As an example, Fig. 1 shows a view of the simulation domain along with 50 electron trajectories. Results presented below are obtained from the analysis of 10000 electron trajectories.

Electron trajectories can be analytically recovered in the case $\phi_{d}=0$, i.e., without the electric field. The equation of motion is solved for one electron in Cartesian coordinates with $\mathbf{B}$ oriented along the $\mathbf{x}$ axis. The condition for the electron to be recollected, $r \leq r_{d}$, is equivalent to, after some straightforward algebra, the existence of at least one root to the function

$$
R(t)=A t^{2}+B t+C\left(\cos \omega_{e} t-1\right)+D \sin \omega_{e} t
$$

where

$$
\begin{aligned}
& A=\omega_{e} v_{x, 0}^{2} / 2, \quad B=\omega_{e} x_{0} v_{x, 0}, \\
& C=y_{0} v_{z, 0}-z_{0} v_{y, 0}-\left(v_{y, 0}^{2}+v_{z, 0}^{2}\right) / \omega_{e}, \\
& D=y_{0} v_{y, 0}+z_{0} v_{z, 0} .
\end{aligned}
$$

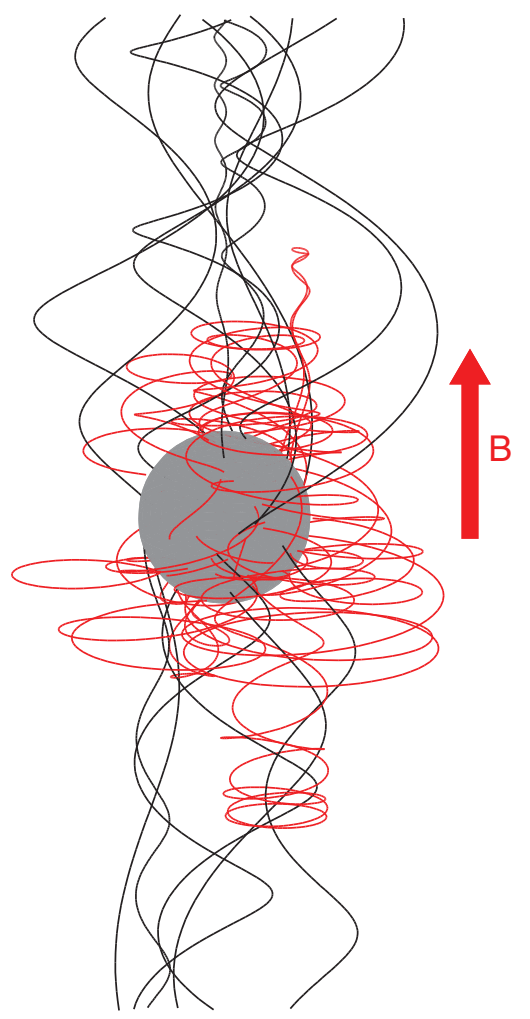

FIG. 1. 50 emitted electron trajectories around a dust grain (grey disc) with $r_{d}=1 \mu \mathrm{m}, e \phi_{d} / T_{e}=0.02$ and $T_{d}=6000 \mathrm{~K}$. Trajectories are plotted in red for recollected electrons and in black for escaped electrons. $\mathbf{B}$ is oriented along the red arrow. In this case, the effective emission yield is $\delta_{\mathrm{E}, \mathrm{B}}$ $=46.7 \%$. 
The function $R$ has a minimum at $t=\tau>0$ and it is obvious that an electron emitted from position $\mathbf{x}_{0}=\left(x_{0}, y_{0}\right.$, $\left.z_{0}\right)$ with velocity $\mathbf{v}_{0}=\left(v_{x, 0}, v_{y, 0}, v_{z, 0}\right)$ will be recollected if $R(\tau) \leq 0$, i.e.,

$$
\delta_{\mathrm{E}, \mathrm{B}}\left(\mathbf{x}_{0}, \mathbf{v}_{0}\right)= \begin{cases}0 & \text { if } R(\tau) \leq 0 \\ 1 & \text { if } R(\tau)>0\end{cases}
$$

Thus, the effective emission yield, defined as the number of emitted electrons that escape the grain over the whole emitted electron population, is obtained by integrating $\delta_{\mathrm{E}, \mathrm{B}}$ $\left(\mathbf{x}_{0}, \mathbf{v}_{0}\right)$ over the initial position and velocity distribution functions of emitted electrons named $f_{x}$ and $f_{v}$, respectively,

$$
\delta_{\mathrm{E}, \mathrm{B}}\left(\phi_{d}=0\right)=\int \delta_{\mathrm{E}, \mathrm{B}}\left(\mathbf{x}_{0}, \mathbf{v}_{0}\right) f_{x}\left(\mathbf{x}_{0}\right) f_{v}\left(\mathbf{v}_{0}\right) d \mathbf{x}_{0} d \mathbf{v}_{0} .
$$

The method used is similar to that of Ref. 15, but in the case of a spherical emitting body, it can be used whenever the sheath electric field effects can be neglected. $\delta_{\mathrm{E}, \mathrm{B}}$ from Eq. (5) is plotted in Figs. 2 and 3(b) as black lines in the case of THE (distribution functions evoked above) and is in good agreement with the Monte Carlo results.

The time evolution of the fraction of promptly recollected electrons (i.e., $1-\delta_{\mathrm{E}, \mathrm{B}}$ ) is plotted in Fig. 2 for two values of dust normalized potential chosen to be $e \phi_{d} / T_{e}=0$ and 0.02 . The time is normalized to the inverse of the electron gyrofrequency $\omega_{e}=e B / m_{e}$.

As expected, higher recollection occurs on positively charged grains due to the attracting electric field. Two distinct phases can be identified. (i) In the order of one period of gyration $\left(t \sim \omega_{e}^{-1}\right)$, the recollection quickly increases in both cases due to the gyration motion. This will be labeled as magnetic recollection. (ii) Electrons that managed to complete at least one gyromotion $\left(t>\right.$ few $\left.\omega_{e}^{-1}\right)$ escape the uncharged grain while some low energy electrons are still recollected by the positively charged one. This will be referred to as electrostatic recollection. Electrons are more likely to undergo electrostatic recollection if they are emitted

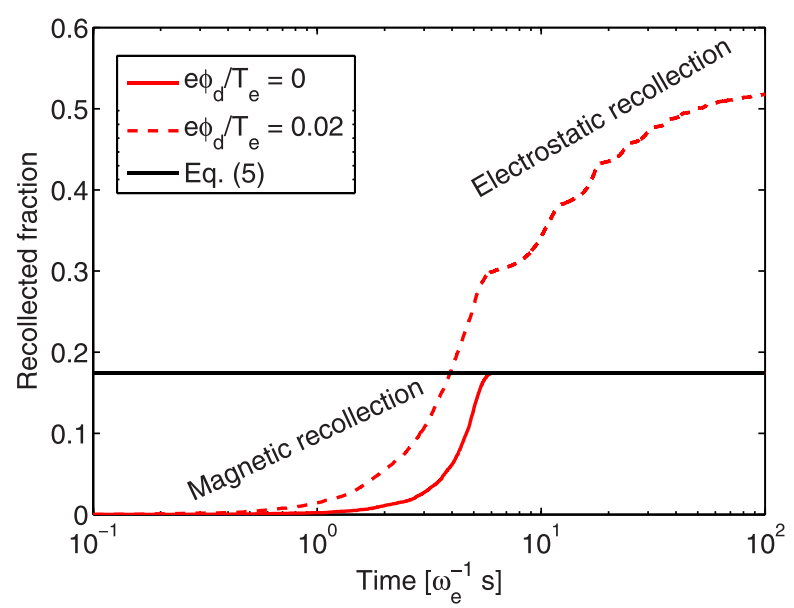

FIG. 2. Time evolution of the fraction of recollected electrons for $r_{d}=1 \mu \mathrm{m}$, $T_{d}=6000 \mathrm{~K}$, and two values of dust potential: $e \phi_{d} / T_{e}=0$ and 0.02 . The analytical recollected fraction in the case $\phi_{d}=0$ computed from Eq. (5) is plotted as a black solid line. The final value of the recollected fraction in the case $e \phi_{d} / T_{e}=0.02$ is $52.3 \%$.
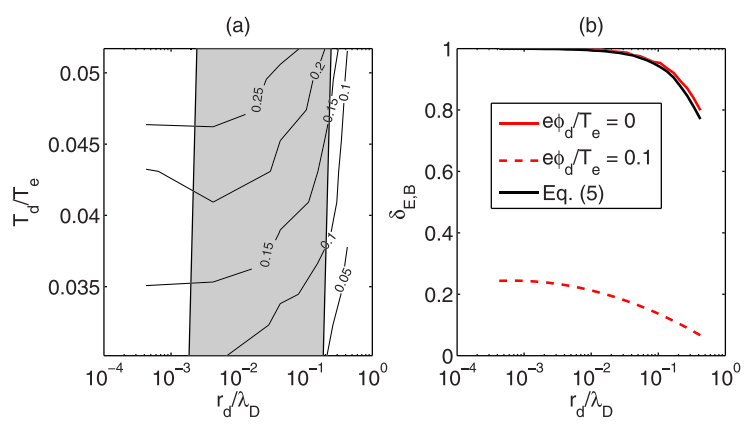

FIG. 3. Effective emission yield $\delta_{\mathrm{E}, \mathrm{B}}$ presented as: (a) contours against $T_{d} / T_{e}$ and $r_{d} / \lambda_{D}$ for $e \phi_{d} / T_{e}=0.1$ with the region corresponding to magnetized emitted electrons in grey and (b) plots against $r_{d} / \lambda_{D}$ for $T_{d}=5000 \mathrm{~K}$ and $e \phi_{d} / T_{e}=0$ and 0.1 in red along with the solution from Eq. (5) in black.

at a grazing angle rather than perpendicularly to the dust surface, whereas magnetic recollection preferentially occurs if electrons are emitted perpendicularly to the magnetic field and from regions where the magnetic field makes a grazing angle with the dust surface, as can be seen in Fig. 1. This can be intuitively understood since, in these areas, the velocity of an emitted electron is mostly perpendicular to the magnetic field, leading to it being reflected back to the surface of the grain. This was also shown in studies performed on planar surfaces (see Ref. 14 and references therein).

Figure 3 shows the Monte Carlo simulation results in terms of effective emission yield $\delta_{\mathrm{E}, \mathrm{B}}$ for different values of dust temperature and potential. The two phenomena cannot be decoupled since $\mathbf{E}$ also plays an important role in the first gyration motion of the electron, and thus on the magnetic recollection.

Contours of $\delta_{\mathrm{E}, \mathrm{B}}$ are plotted in Fig. 3(a) for a fixed value of the dust potential, $e \phi_{d} / T_{e}=0.1$, and varying dust temperature and size. The grey shaded area is the region where the gyroradius of secondary electrons is comparable to $r_{d}$, i.e., $10^{-2} \leq r_{d} / \rho_{\text {Lse }} \lesssim 1$. When $r_{d} \ll \rho_{\text {Lse }}$, secondary electrons are not magnetized and $\delta_{\mathrm{E}, \mathrm{B}}$ increases with $T_{d}$ since electrons have more energy to escape the sheath potential drop. When $r_{d} \gtrsim \rho_{L s e}, \delta_{\mathrm{E}, \mathrm{B}}$ becomes independent on $r_{d}$ since the scale length of an electron trajectory remains small with respect to the dust curvature. In Fig. 3(b), $\delta_{\mathrm{E}, \mathrm{B}}$ is plotted against the dust size for different values of the dust potential and $T_{d}=5000 \mathrm{~K}$, which corresponds to $\rho_{L s e} / \lambda_{D} \approx 0.22$. When $r_{d} / \lambda_{D} \ll 0.1$, emitted electrons are unmagnetized and only electrostatic recollection is observed on the positively charged grain $\left(e \phi_{d} / T_{e}=0.1\right)$ while $\delta_{\mathrm{E}, \mathrm{B}} \approx 1$ for the uncharged grain. As expected, the effective emission yield decreases as $r_{d}$ gets to the order of $\rho_{\text {Lse }}$ due to magnetic recollection.

The effect of the prompt recollection on any electron emission process satisfying the condition $\rho_{L s e} \ll \rho_{L e}$ can be estimated using the method presented earlier, given the distribution functions for their velocity and angle of emission. The overall yield of a given electron emission process including prompt recollection effects can be recovered by multiplying $\delta_{\mathrm{E}, \mathrm{B}}$ by the emission yield of the process considered, i.e., the number of emitted electrons per incident electron. In the case of SEE, one should use the Chung-Everhart distribution function ${ }^{20}$ and an angle distribution that follows 
a cosine of the angle with the normal to the surface. This is because SEE electrons are created in the bulk with random velocity orientation and their probability to reach the dust surface is inversely proportional to the length of their path. This work is easily performed and redundant results will not be presented here.

Even though it was recently shown that the dust lifetime is roughly independent on its global electron emission yield in the regime $\lambda_{D}, \rho_{L e} \ll r_{d} \lesssim \rho_{L i}{ }^{21}$ it is still important to quantitatively estimate the electron emission in order to model the dust transport accurately. Moreover, the correct depiction of the dust potential is crucial in the case of small grains where the OML theory still applies..$^{3,4,22-24}$ In the OML framework, reduction in the electron emission yield should lead to more negative (or less positive) values of the dust charge, inducing a change in the heat fluxes balance. Mainly, electron heating should be reduced along with electron emission cooling, whilst ion heating should increase. Although this result can be used for any dusty plasma application, we will focus on fusion in the following.

Figure 4 shows the dependency of a $\mathrm{W}$ dust floating potential on its temperature $T_{d}$, computed by solving the current balance using the usual OML expressions for collected currents and taking into account both SEE and THE. In the first case (dashed black line), only electrostatic recollection is accounted for, using the expressions implemented in the MIGRAINe code, ${ }^{3}$ whilst in the second one (solid red line), $\delta_{\mathrm{E}, \mathrm{B}}$ is incorporated for both emission processes. The SEE current was computed from the Young-Dekker formula ${ }^{25}$ and integrated over the primary electrons energy distribution function (assumed to be Maxwellian) and the impact angle distribution. The Richardson-Dushman formula was used for the THE current. ${ }^{26}$ Both currents were then multiplied by the yield $\delta_{\mathrm{E}, \mathrm{B}}$ computed for each emission process in the form of tabulated values. The background density used and displayed in Table I ensures that $r_{d} \ll \lambda_{D}$. Yet, it has been shown that OML is accurate up to $r_{d} \sim 10 \lambda_{D}{ }^{24}$ Thus, herein presented results hold for denser plasmas, e.g., up to $\sim 10^{22} \mathrm{~m}^{-3}$ for micron size dust.

Differences are observed for $\phi_{d} \geq 0$, where the floating potential is reduced by up to $40 \%$ as $T_{d}$ approaches $6000 \mathrm{~K}$.

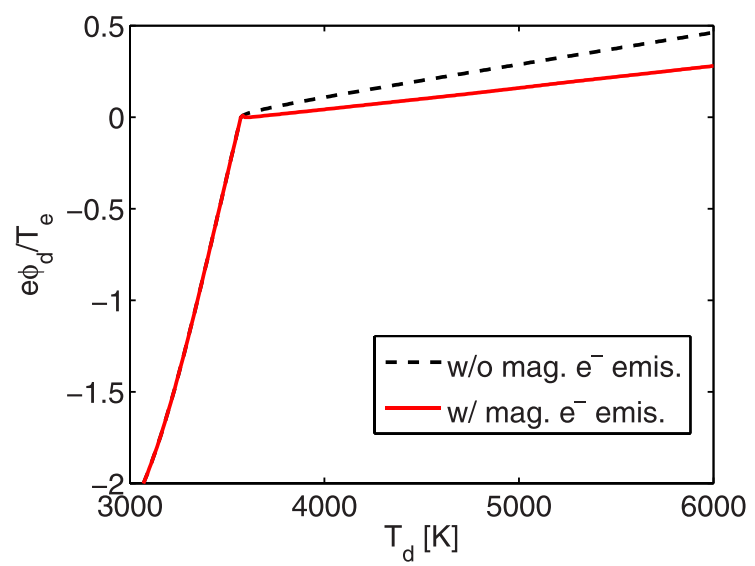

FIG. 4. W dust floating potential as a function of its temperature computed by solving the current balance accounting for electrostatic recollection only in dashed black and both electrostatic and magnetic recollection in solid red. The dust radius is fixed at $r_{d}=1 \mu \mathrm{m}$.

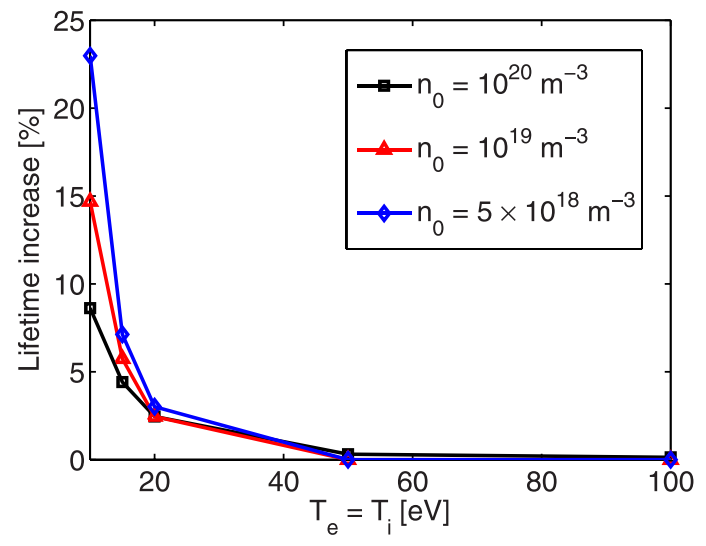

FIG. 5. W dust lifetime in a pure D homogeneous plasma with varying electron/ion temperature and density. The initial dust radius is $r_{d}=1 \mu \mathrm{m}$.

The values of $\phi_{d}$ computed with the inclusion of magnetic recollection are consistent with the range described earlier $\left(0 \leq e \phi_{d} / T_{e} \leq 0.2\right)$ and for which potential well effects can be neglected.

Incorporating magnetic recollection effects in the dust transport code DUMBO confirmed that the dust lifetime can be increased due to reduced electron heating at a high dust temperature. In Fig. 5, a W dust grain with an initial radius of $1 \mu \mathrm{m}$ was simulated in a plasma with varying temperature (keeping $T_{e}=T_{i}$ ) and density. The most significant lifetime increase is observed in the coldest and least dense plasma $\left(T_{e}=10 \mathrm{eV}\right.$ and $n_{0}=5 \times 10^{18} \mathrm{~m}^{-3}$ ) where it reaches $23 \%$, which is not negligible yet not sufficient to explain lifetime discrepancies between dust transport simulations and camera observations of injected dust. ${ }^{2,27}$

In conclusion, the prompt recollection of emitted electrons on a spherical dust grain due to the presence of a magnetic field has been investigated through Monte Carlo simulations. The gyration motion of electrons leads to a reduction in the emission yield. It was shown that dust charging can be significantly affected by this effect, especially at a high dust temperature (where the thermionic emission is strong and the dust is positively charged). In this regime, the dust floating potential is reduced. Consequently, dust heating is also reduced and the dust lifetime increases at temperatures and densities relevant for tokamak SOL plasmas. When the sheath electric field can be neglected, Eq. (5) can be used to estimate prompt recollection. Results presented in the fusion-related context can be applied to any dusty plasma application where magnetic fields are present.

In our Monte Carlo simulations, the effect of the electron population on the potential profile is not accounted for. A self-consistent model including studies on electron emission with non-monotonic potential profiles will be assessed in future works.

S.A.K., B.K., and N.N. were supported by the A*MIDEX Project (No. ANR-11-IDEX-0001-02) funded by the French Government "Investissements d'Avenir" program managed by the French National Research Agency (ANR).

${ }^{1}$ S. I. Krasheninnikov, R. D. Smirnov, and D. L. Rudakov, Plasma Phys. Controlled Fusion 53, 083001 (2011). 
${ }^{2}$ A. Autricque, S. Hong, N. Fedorczak, S. Son, H. Lee, I. Song, W. Choe, and C. Grisolia, in Proceedings of the 22nd International Conference on Plasma Surface Interactions, 2016 [Nucl. Mater. Energy 12, 599 (2017).

${ }^{3}$ L. Vignitchouk, P. Tolias, and S. Ratynskaia, Plasma Phys. Controlled Fusion 56, 095005 (2014).

${ }^{4}$ R. D. Smirnov, A. Y. Pigarov, M. Rosenberg, S. I. Krasheninnikov, and D. A. Mendis, Plasma Phys. Controlled Fusion 49, 347 (2007).

${ }^{5}$ M. Bacharis, M. Coppins, and J. E. Allen, Phys. Plasmas 17, 042505 (2010).

${ }^{6}$ E. Lazzaro, I. Proverbio, F. Nespoli, S. Ratynskaia, C. Castaldo, U. deAngelis, M. DeAngeli, J.-P. Banon, and L. Vignitchouk, Plasma Phys. Controlled Fusion 54, 124043 (2012).

${ }^{7}$ H. M. Mott-Smith and I. Langmuir, Phys. Rev. 28, 727 (1926).

${ }^{8}$ J. E. Allen, Phys. Scr. 45, 497 (1992).

${ }^{9}$ V. Fortov, A. Ivlev, S. Khrapak, A. Khrapak, and G. Morfill, Phys. Rep. 421, 1 (2005).

${ }^{10}$ J. D. Martin, "Theory and simulation of dust in tokamak plasmas," $\mathrm{Ph} . \mathrm{D}$. thesis (Imperial College of London, London, 2006).

${ }^{11}$ L. Patacchini, I. H. Hutchinson, and G. Lapenta, Phys. Plasmas 14, 062111 (2007)

${ }^{12}$ G. L. Delzanno, A. Bruno, G. Sorasio, and G. Lapenta, Phys. Plasmas 062102 (2005).

${ }^{13}$ J. D. Martin, M. Bacharis, M. Coppins, G. F. Counsell, and J. E. Allen, EPL (Europhys. Lett.) 83, 65001 (2008).
${ }^{14}$ M. Komm, S. Ratynskaia, P. Tolias, J. Cavalier, R. Dejarnac, J. P. Gunn, and A. Podolnik, Plasma Phys. Controlled Fusion 59, 094002 (2017).

${ }^{15}$ D. Tskhakaya and S. Kuhn, Contributions to Plasma Phys. 40, 484 (2000).

${ }^{16} \mathrm{Y}$. Igitkhanov and G. Janeschitz, in 14th International Conference on Plasma-Surface Interactions in Controlled Fusion Devices [J. Nucl. Mater. 290, 99 (2001)].

${ }^{17}$ I. E. Berezina and I. V. Tsvetkov, J. Phys.: Conf. Ser. 516, 012025 (2014).

${ }^{18}$ S. Takamura, M. Y. Ye, T. Kuwabara, and N. Ohno, Phys. Plasmas 5, 2151 (1998).

${ }^{19}$ G. L. Delzanno and X.-Z. Tang, Phys. Rev. Lett. 113, 035002 (2014).

${ }^{20}$ M. S. Chung and T. E. Everhart, J. Appl. Phys. 45, 707 (1974).

${ }^{21}$ L. Vignitchouk, S. Ratynskaia, and P. Tolias, Plasma Phys. Controlled Fusion 59, 104002 (2017).

${ }^{22}$ G. L. Delzanno, G. Lapenta, and M. Rosenberg, Phys. Rev. Lett. 92, 035002 (2004)

${ }^{23}$ G. L. Delzanno and X. Tang, Phys. Plasmas 21, 022502 (2014).

${ }^{24}$ G. L. Delzanno and X.-Z. Tang, Phys. Plasmas 22, 113703 (2015).

${ }^{25}$ P. Tolias, Plasma Phys. Controlled Fusion 56, 123002 (2014).

${ }^{26}$ S. Dushman, Rev. Mod. Phys. 2, 381 (1930).

${ }^{27}$ A. Shalpegin, L. Vignitchouk, I. Erofeev, F. Brochard, A. Litnovsky, S. Bozhenkov, I. Bykov, N. den Harder, and G. Sergienko, Plasma Phys. Controlled Fusion 57, 125017 (2015). 